\title{
Large chest wall fibromatosis with challenging treatment plan
}

\author{
Rashi Agrawal, Prekshi Choudhary, Arun Kumar Goel, Vaishali Zamre, Sandeep Agarwal, Dinesh Singh \\ Department of Radiation Oncology, Surgical Oncology, Max Super Speciality Hospital, Vaishali, Ghaziabad, Uttar Pradesh 201012, Delhi NCR, India.
}

Correspondence to: Dr. Rashi Agrawal, Department of Radiation Oncology, Surgical Oncology, Max Super Speciality Hospital, W3, sector 1, Vaishali, Ghaziabad, Uttar Pradesh 201012, Delhi NCR, India. E-mail: Drrashi.ag@gmail.com

How to cite this article: Agrawal R, Choudhary P, Goel AK, Zamre V, Agarwal S, Singh D. Large chest wall fibromatosis with challenging treatment plan. J Cancer Metastasis Treat 2017;3:139-43.

\section{Article history: \\ Received: 03-01-2017 \\ Accepted: 28-04-2017 \\ Published: 21-07-2017}

Key words:

Chest wall fibromatosis,

recurrence,

radiotherapy

\begin{abstract}
The primary mode of treatment for desmoid tumors is surgical excision. However, high recurrence rates $(39-79 \%)$ have been reported when surgery is used alone. The role of adjuvant radiotherapy after surgical resection of primary disease is controversial and should be based on a balanced discussion of potential morbidity from radiotherapy and local recurrence. In this patient, the maximum dimension of tumor was $21 \mathrm{~cm}$. This is a larger chest wall fibromatosis than has been reported thus far, to the best of our knowledge. In this case, post-operative margins were free, but in view of the large initial tumor size and potential morbidity in case of any future locoregional recurrence, post-operative adjuvant external beam radiation was delivered. An image guided intensity modulated radiotherapy technique was chosen to spare adjacent breast and lung parenchyma, and tolerance of these structures was well respected. This case provides insight into this treatment approach.
\end{abstract}

\section{INTRODUCTION}

Desmoid tumors, also termed as aggressive fibromatosis, are heterogeneous, benign tumors that originate from deep musculoaponeurotic structures. These rare tumors account for approximately 0.03$0.1 \%$ of all solid tumors and $3.6 \%$ of all fibrous tissue neoplasms. ${ }^{[1]}$ Fibromatoses of the chest wall are rare and only represent $10 \%$ to $20 \%$ of all fibromatoses. ${ }^{[2,3]}$

These neoplasms can display local infiltrative growth but due to their benign nature they do not metastasize.$^{[4]}$ Desmoid tumors are non-encapsulated and tend to extend along fascial planes. They also have the potential to erode bone and surrounding blood vessels or nerves.

In this report, we present the case of a patient with a large chest wall aggressive fibromatosis, $21 \mathrm{~cm}$ in maximum dimension, who was treated with surgery followed by adjuvant modern image guided radiation therapy. Standard protocols and recent trends for the treatment of desmoid tumors are also discussed.

\section{CASE REPORT}

A 24-year-old female presented with complaints of pain in the right lower chest for 2.5 months and 
breathlessness for 2 months. Chest X-ray (PA view) reported dense homogeneity over the right middle and lower zones. A computed tomography (CT) scan of thorax and abdomen with contrast showed a large pleural based mass of approximately $12 \mathrm{~cm} \times 13 \mathrm{~cm}$ $\times 19 \mathrm{~cm}$ in the right thoracic cavity, probably arising from right chest wall, extending into the mediastinum, with smooth indentation on pericardium and superior vena cava with no evidence of rib destruction [Figures 1 and 2]. Core needle biopsy of the mass showed a benign spindle cell tumor.

She underwent excision of the tumor along with a portion of ribs and intercostal muscle under general anesthesia on June 11, 2013. Intraoperative findings confirmed a large, firm mass in the right chest wall arising from anterior parts of the lower ribs. Lung, diaphragm and mediastinal structures were not infiltrated. Repair of the chest wall defect was done using double layer polypropylene mesh. Postoperative histopathology reported a benign spindle cell tumor of $21 \mathrm{~cm} \times 15 \mathrm{~cm} \times 5.5 \mathrm{~cm}$ with negative margins. On gross examination there was a single soft tissue piece with attached bone and skeletal muscle. Immunohistochemistry reports revealed tumor cells focally positive for SMA and negative for S-100 and CD 34, suggestive of extra abdominal fibromatosis.

The patient was prepared for postoperative adjuvant external beam radiation to the chest wall (postoperative bed) in view of the unusually large primary neoplasm and increased risk of recurrence. For immobilization, both thermoplastic mould and VACLOC of chest were made. The patient was kept in supine position with both arms abducted alongside of the head. For CT simulation, a radiation technologist accompanied the patient; the same. Positioning as during immobilization was followed. During CT simulation radio opaque markers were placed over the scar mark. A CT scan of the area of interest was taken using $2 \mathrm{~mm}$ slice thickness without intravenous contrast. The radiotherapy equipment used was dualenergy linear accelerator (Clinac iX, Varian Oncology System) incorporating asymmetric $X$ and $Y$ collimators, 120-leaf millenium-multileaf collimator, amorphous silicon-based electronic portal imaging, kilovoltage cone beam CT scanner, 3D beam planning computer workstation (Eclipse TPS ver 8.6.17) and networking (ARIA network).

After thorough discussions with the surgeon, radiologist, and based on preoperative images, contouring of the postoperative bed (clinical target volume) was done. All the organs at risk were contoured according to RTOG guidelines. Radiotherapy doses of $50.4 \mathrm{~Gy}$ in
28 fractions was delivered at the rate of $1.8 \mathrm{~Gy}$ per fraction, 5 fractions per week for 5 weeks to clinical target volume (CTV) by image guided radiotherapy technique. The doses delivered to CTV in the axial, coronal and saggital sections are represented in Figures 3-5. Adjacent normal structures (right lung, heart, right breast, liver) were given dose constraints. We achieved a volume of 20 Gy (V20) as follows,

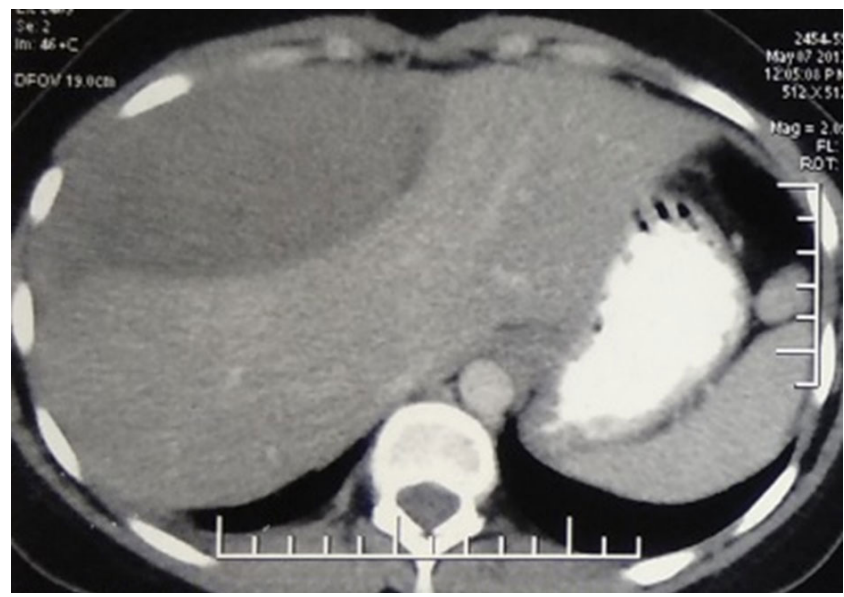

Figure 1: Axial image of computed tomography chest at level of liver

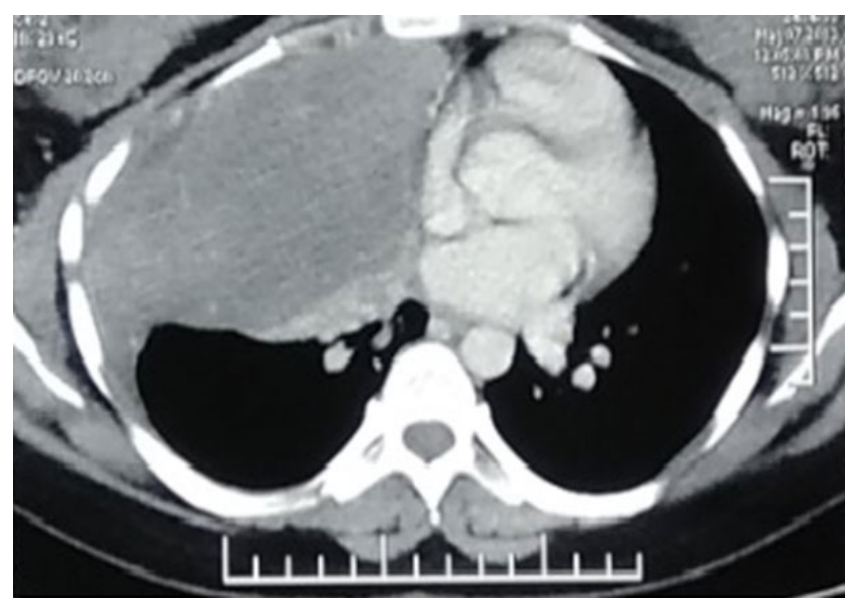

Figure 2: Axial image of computed tomography chest at level of heart

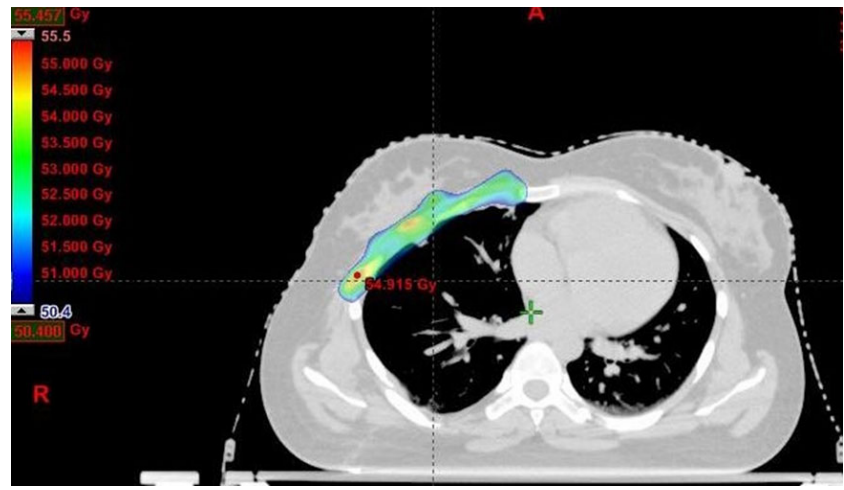

Figure 3: Axial image of radiation dose distribution 


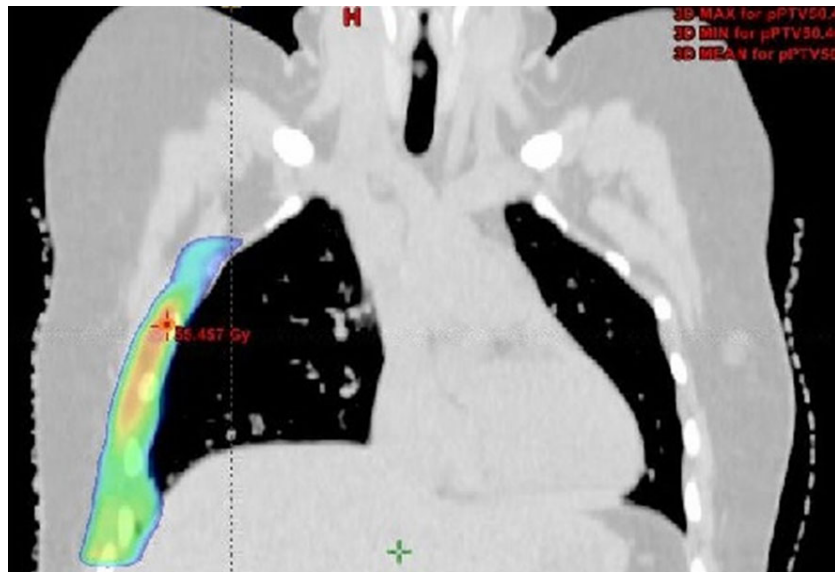

Figure 4: Coronal image of radiation dose distribution

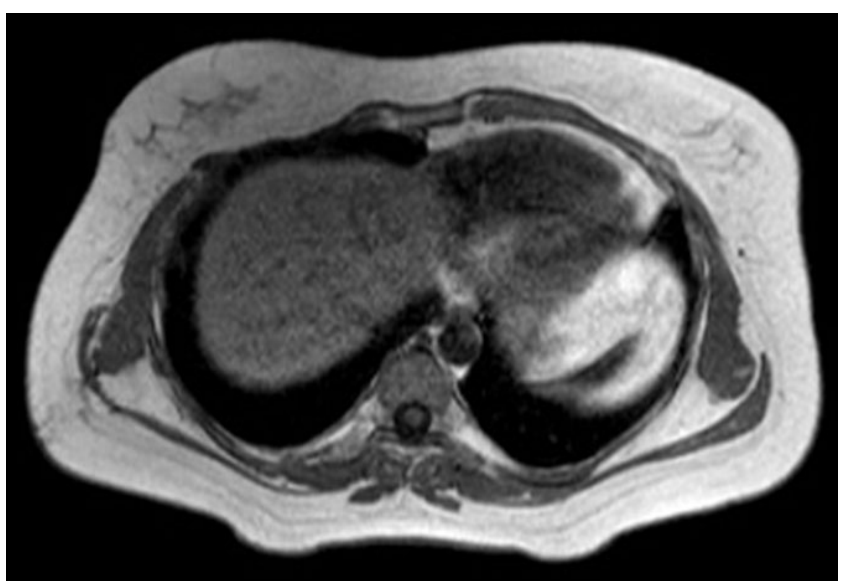

Figure 6: Axial image of magnetic resonance imaging chest at 3.5 years

$21 \%$ for right lung, $16.6 \%$ for liver and $1.8 \%$ for heart. Maximum dose to left lung was $10.6 \mathrm{~Gy}$. The patient tolerated the treatment well and did not report any toxicity. She is in regular follow-up since then. After a follow-up of 3.5 years, the patient is symptomatically free and clinical and radiological examination reveal results within normal limits as did a recent magnetic resonance imaging of the chest [Figure 6]. Presently the patient is not experiencing any skin fibrosis, pulmonary and cardiac toxicity. Bilateral breasts are also normal.

\section{DISCUSSION}

Desmoid tumors are an aggressive fibroblastic proliferation of well circumscribed, locally invasive, differentiated fibrous tissue. On gross examination, they appear as dense, rubbery gray-white masses. ${ }^{[5]}$ The most common locations for desmoid tumors include the shoulder, chest wall, and thigh. Males and females of all ages can be affected by desmoid tumors, but a propensity for fertile women has been noted by many authors. ${ }^{\left[{ }^{[6]}\right.}$ Possible risk factors include
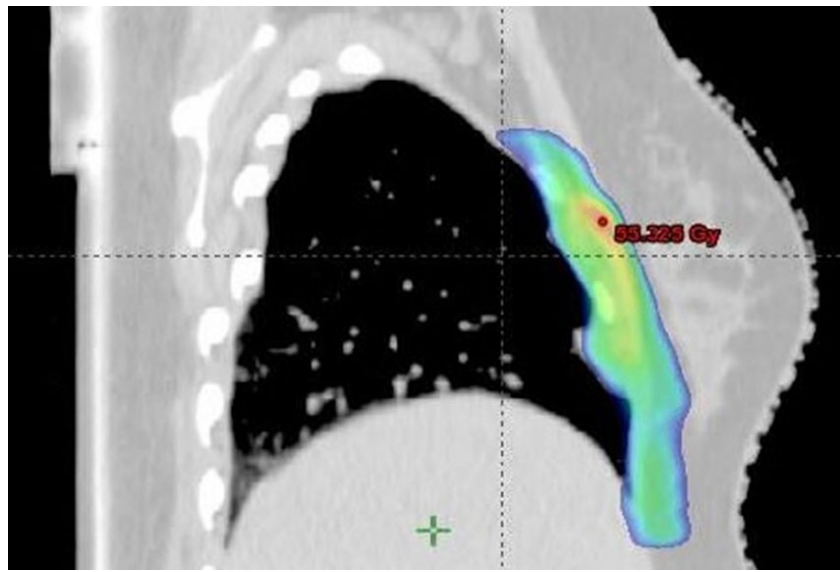

Figure 5: Sagittal image of radiation dose distribution

estrogen status, pregnancy, physical and surgical trauma, radiation, skeletal abnormalities, and genetic determinants. ${ }^{[7]}$ Our patient was of a fertile age group but not pregnant.

Fibromatosis of the chest wall usually presents in the form of a tumor of various sizes which is often large. In the literature, the size of tumors has been reported as being from $5 \mathrm{~cm}$ to $10 \mathrm{~cm}$ and is rarely larger than $20 \mathrm{~cm}$. In the series by Kabiri et al. ${ }^{[3]}$ tumor size varied from $2 \mathrm{~cm}$ to $13 \mathrm{~cm}$ with a mean of $6 \mathrm{~cm}$. In another series, the average tumor was $8.75 \mathrm{~cm}{ }^{\left[{ }^{[8]}\right.}$ In our patient maximum dimension was $21 \mathrm{~cm}$. This is a larger chest wall fibromatosis than has been reported thus far. This fibromatosis has only become symptomatic due to the mechanical compression of neighboring organs. ${ }^{[9]}$ In our patient, the tumor was compressing a lung, hence, the patient was having breathlessness.

The primary mode of treatment for desmoid tumors is surgical excision. However, surgery alone has resulted in high recurrence rates. ${ }^{[10]}$ The goal of surgical excision is gross total resection with negative margins. Recurrence of desmoid tumors may be related to the age of the patient, the site of tumor, and the initial form of treatment. In a retrospective study of 142 patients by Fiore et al., ${ }^{[1]]}$ it was found that larger tumors and those tumors located on the trunk were associated with a higher risk of recurrence. Local control rates have been reported to be a function of tumor location, ability to obtain negative margins, and adjuvant therapy. In cases of RO resection, a patient can be kept on observation but post-operative radiation is to be considered for larger tumors as it reduces the risk of loco-regional recurrence. For R1 resection (microscopic positive margins), either re-surgery or high dose radiation (66-70 Gy) is recommended. Postoperative radiotherapy reduces the risk of recurrence in patients with positive margins, improves local 
Table 1: Chest wall fibromatoses treatment and results

\begin{tabular}{lccc}
\hline Study & Number of patients & Treatment & Result \\
\hline Zehani-Kassar et al. ${ }^{[8]}$ & 6 & Surgery & Recurrence in 1 patient \\
Abbas et al..$^{18]}$ & 53 & Surgery + radiotherapy & $37.5 \%$ recurrence probability \\
Varghese et al..$^{[14]}$ & 1 & Surgery & No recurrence \\
Sakamoto et al. ${ }^{[19]}$ & 1 & Surgery + radiotherapy & At 15 months: no recurrence \\
\hline
\end{tabular}

control and improves progression-free survival. For R2 resection (macroscopic positive margins), high dose radiation followed by boost (70-76 Gy) has been recommended.

Systemic therapy using NSAIDs, hormonal/biological agents, or cytotoxic drugs also plays an important role in patients with desmoid tumors. ${ }^{[12]}$ In a prospective study, tamoxifen along with sulindac has been used for disease stabilization in recurrent or progressive disease after surgery. Interferon-alpha, toremifene and doxorubicin, vinblastin and methotrexate-based chemotherapy and tyrosine kinase inhibitors (imatinib, sorafinib) have also been used in recurrent progressive tumors after surgery.

The overall rate of recurrence ranges between $25 \%$ and $75 \%$. There is a huge variation in rate in the literature. ${ }^{[9,13]}$ Although survival at 5 years is nearly $93 \%$, the probability of recurrence is an estimated 29\%. ${ }^{[14]}$ Even though these tumors do not metastasize, they can result in significant morbidity and death from locoregional invasion. ${ }^{[15]}$ The role of adjuvant radiotherapy after surgical resection of primary disease is controversial and should be based on a balanced discussion of potential morbidity from radiotherapy and recurrence. The local control of desmoid tumor in the adjuvant setting is excellent, with total doses ranging from 50-60 Gy, with acceptable morbidity. Margin status is one of the most important predictor of recurrence after surgery in desmoid tumors. If a future local recurrence would incur even greater morbidity or would be potentially unresectable, then adding adjuvant radiotherapy would be reasonable. The benefit of radiotherapy has been claimed in several reports. In particular, a review by Nuyttens et al. ${ }^{[16]}$ including more than 20 retrospective studies focusing on the role of the combination (surgery and radiotherapy), showed that surgery plus radiotherapy or radiotherapy alone could obtain a better local control rate $(75 \%$ and $78 \%$, respectively) compared with surgery alone $(61 \%)$. However, this is an extremely debated topic. According to Gronchi et al., ${ }^{[17]}$ these tumors represent a relatively benign condition and most of the patients are young; hence the authors suggest radiotherapy only for documented progressive disease and in absence of other alternatives. Table 1 shows some literature on chest wall fibromatoses with their treatment and results.

In our patient, the clinical history was short, covering only two and half months. This patient was symptomatic (breathlessness along with pain). Tumor size was extremely large (maximum dimension $21 \mathrm{~cm}$ ). The operating surgeon was also in favor of postoperative radiation due to potential morbidity in case of future locoregional recurrence.

Hence in spite of the post-operative margins being free, adjuvant external beam radiation was planned. Radiation by conventional techniques leads to increased doses to adjacent normal structures (lungs and breast in this case), which may lead to late complications in the form of fibrosis or secondary malignancies. However, with the use of newer techniques, it is possible to give homogenous dose distribution to the target volume, while keeping the dose to critical and normal structures within normal range. In our case, we chose image-guided intensity modulated radiotherapy technique to spare maximum normal tissues (adjacent breast and lung parenchyma). Tolerance of lungs and heart was well respected. This case provides valuable insights into potential treatment approaches in such a rare presentation.

\section{Authors' contributions}

Writing and conceptualization of this work: R. Agrawal, P. Choudhary

Supervising the work: V. Zamre, A.K. Goel, S. Agarwal, D. Singh

\section{Financial support and sponsorship}

None.

\section{Conflicts of interest}

There are no conflicts of interest.

\section{Patient consent}

Patient's consent was obtained as per institutional policy.

\section{Ethics approval}

The ethics approval was obtained from the institutional ethical committee for preparation and publication of this paper. 


\section{REFERENCES}

1. Pereyo NG, Heimer WL 2nd. Extraabdominal desmoid tumor. $J$ Am Acad Dermatol 1996;34:352-6.

2. Ibrahim M, Sandogji H, Allam A. Huge intrathoracic desmoids tumor. Ann Thorac Med 2009;4:146-8.

3. Kabiri EH, Al Aziz S, El Maslout A, Benosman A. Desmoid tumors of the chest wall. Eur J Cardiothorac Surg 2001;19:580-3.

4. Acker JC, Bossen EH, Halperin EC. The management of desmoid tumors. Int J Radiat Oncol Biol Phys 1993;26:851-8.

5. Canale ST. Campbell's operative orthopaedics. 9th ed. St. Louis, MO: Mosby, Inc; 1998. p. 744-6.

6. Reitamo JJ, Scheinin TM, Häyry P. The desmoid syndrome. New aspects in the cause, pathogenesis and treatment of the desmoid tumor. Am J Surg 1986;151:230-7.

7. Lucas DR, Shroyer KR, McCarthy PJ, Markham NE, Fujita M, Enomoto TE. Desmoid tumor is a clonal cellular proliferation: PCR amplification of HUMARA for analysis of patterns of X-chromosome inactivation. Am J Surg Pathol 1997;21:306-11.

8. Zehani-Kassar A, Ayadi-Kaddour A, Marghli A, Ridene I, Daghfous H, Kilani T, El Mezni F. Desmoid-type chest wall fibromatosis. A six cases series. Orthop Traumatol Surg Res 2011;97:102-7.

9. Zisis C, Dountsis A, Nikolaides A, Dahabreh J. Desmoid tumors of the chest wall. Asian Cardiovasc Thorac Ann 2006;14:359-62.

10. Goy BW, Lee SP, Eilber F, Dorey F, Eckardt J, Fu YS, Juillard GJ, Selch MT. The role of adjuvant radiotherapy in the treatment of resectable desmoid tumors. Int $J$ Radiat Oncol Biol Phys 1997;39:659-65.

11. Fiore M, Rimareix F, Mariani L, Domont J, Collini P, Le Péchoux C, Casali PG, Le Cesne A, Gronchi A, Bonvalot S. Desmoid-type fibromatosis: a front-line conservative approach to select patients for surgical treatment. Ann Surg Oncol 2009;16:2587-93.

12. Janinis J, Patriki M, Vini L, Aravantinos G, Whelan JS. The pharmacological treatment of aggressive fibromatosis: a systematic review. Ann Oncol 2003;14:181-90.

13. Allen PJ, Shriver CD. Desmoid tumors of the chest wall. Semin Thorac Cardiovasc Surg 1999;11:264-9.

14. Varghese TK Jr, Gupta R, Yeldandi AV, Sundaresan SR. Desmoid tumor of the chest wall with pleural involvement. Ann Thorac Surg 2003;76:937-9.

15. Schlemmer M. Desmoid tumors and deep fibromatoses. Hematol Oncol Clin North Am 2005; 19:565-71, vii-viii.

16. Nuyttens JJ, Rust PF, Thomas CR Jr, Turrisi AT 3rd. Surgery versus radiation therapy for patients with aggressive fibromatosis or desmoid tumors: a comparative review of 22 articles. Cancer 2000;88:1517-23.

17. Gronchi A, Colombo C, Le Péchoux C, Dei Tos AP, Le Cesne A, Marrari A, Penel N, Grignani G, Blay JY, Casali PG, Stoeckle E, Gherlinzoni F, Meeus P, Mussi C, Gouin F, Duffaud F, Fiore M, Bonvalot S; ISG and FSG. Sporadic desmoid-type fibromatosis: a stepwise approach to a non-metastasising neoplasm -- a position paper from the Italian and the French Sarcoma Group. Ann Oncol 2014;25:578-83.

18. Abbas AE, Deschamps C, Cassivi SD, Nichols FC 3rd, Allen MS, Schleck CD, Pairolero PC. Chest-wall desmoid tumors: results of surgical intervention. Ann Thorac Surg 2004;78:1219-23; discussion 1219-23.

19. Sakamoto K, Okita M, Takeuchi K, Nishida S, Mikami Y, Hayashi Y. Desmoid tumor of the chest wall infiltrating into the brachial plexus: report of a resected case. Kyobu Geka 2001;54:160-3. (in Japanese) 